\title{
Does the $D^{-} / D^{+}$production asymmetry decrease at large $x_{F}$ ?
}

\author{
F. Carvalho *, F.O. Durães ${ }^{\dagger}$, F.S. Navarra ${ }^{\ddagger}$, M. Nielsen ${ }^{\S}$ \\ Instituto de Física, Universidade de São Paulo \\ C.P. 66318, 05315-970 São Paulo, SP, Brazil
}

\begin{abstract}
We have applied the meson cloud model (MCM) to calculate the asymmetries in $D$ and $D_{s}$ meson production in high energy $\Sigma^{-}$-nucleus and $\pi^{-}$-nucleus collisions. We find a good agreement with recent data. Our results suggest that the asymmetries may decrease at large $x_{F}$.
\end{abstract}

*e-mail: babi@if.usp.br

†e-mail: fduraes@if.usp.br

${ }^{\ddagger}$--mail: navarra@if.usp.br

§e-mail: mnielsen@if.usp.br 
Several experiments have reported [1] 5] a significant difference between the $x_{F}$ (Feynman momentum) dependence of leading and nonleading charmed mesons $(D)$. Recent data taken by the WA89 collaboration 5 with a $\Sigma^{-}$beam have not only confirmed the asymmetry and the leading particle effect but have also observed this effect in $D_{s}$ and $\Lambda_{c}$ production. An interesting feature of the WA89 data is that they suggest, inspite of very large error bars and poor statistics, that the asymmetry decreases at very large $x_{F}$. More recently, preliminary data from the SELEX collaboration [6], in contrast to all previous experiments, seem to indicate that the asymmetry is smaller and follows a more complicated pattern.

Very soon after the appearence of the first data, it became clear that it was not possible to understand them only with usual perturbative QCD or with the string fragmentation model contained in PYTHIA. Alternative models have been advanced [0,8]. All these models obtain a reasonable description of data, but none of them predicts a decrease in the asymmetry.

The purpose of this letter is to show that in the meson cloud model (MCM) [9] we can reproduce data and accomodate a possible decrease of the asymmetry. The MCM has been very successful in the study of hadron structure [9] and of particle production in high energy soft hadron collisions [10,11]. It has been extended to the charm sector [12].

In the MCM we assume that quantum fluctuations in the projectile play an important role. Both the $\Sigma^{-}$and $\pi^{-}$may be decomposed in a series of Fock states. This series has also been discussed in Ref. [8], where, for example, fluctuations of the type $\left|\pi^{-}\right\rangle=|\bar{u} d \bar{c} c\rangle$ and $\left|\Sigma^{-}\right\rangle=|d d s \bar{c} c\rangle$ were considered. In the MCM we write the Fock decomposition in terms of the equivalent hadronic states $\left|\pi^{-}\right\rangle=\left|D^{0 *} D^{-}\right\rangle$and $\left|\Sigma^{-}\right\rangle=\left|\Xi_{c}^{0} D^{-}\right\rangle$. This expansion contains the "bare" terms (without cloud fluctuations), light states and states containing the produced charmed meson $\left(D\right.$ or $\left.D_{s}\right)$. The latter are, of course very much suppressed but they will be responsible for asymmetries. The "bare" states occur with a higher probability and are responsible for the bulk of charm meson production at low and medium momentum $\left(x_{F} \leq 0.4\right)$, including, for example the perturbative QCD contribution. The cloud states are less frequent fluctuations and contribute to $D$ production in the ways described below. More precisely we shall assume that:

$$
\begin{gathered}
\left|\Sigma^{-}\right\rangle=Z\left[\left|\Sigma_{0}^{-}\right\rangle+\ldots+\left|\Xi_{c}^{0} D^{-}\right\rangle+\left|\Sigma_{c}^{0} D_{s}^{-}\right\rangle\right] \\
\left|\pi^{-}\right\rangle=Z^{\prime}\left[\left|\pi_{0}^{0}\right\rangle+\ldots+\left|D^{0 *} D^{-}\right\rangle\right]
\end{gathered}
$$

where $Z$ and $Z^{\prime}$ are normalization constants, $\left|\Sigma_{0}^{-}\right\rangle$and $\left|\pi_{0}^{0}\right\rangle$ are the "bare" sigma and pion and the "dots" denote possible cloud states $|M B\rangle$ in the $\Sigma^{-}$and $\left|M M^{\prime}\right\rangle$ or $\left|B \bar{B}^{\prime}\right\rangle$ in the $\pi^{-}$. The relative normalization of these states is fixed once the cloud parameters are fixed. We shall first study the reactions induced by the cloud component of the $\Sigma^{-}$. This projectile baryon is thus regarded as being a sum of virtual meson $(M)$-baryon $(B)$ pairs and a $\Sigma^{-}$proton reaction can thus be viewed as a reaction between the "constituent" mesons and baryons of the $\Sigma^{-}$with the target proton.

With a $\Sigma^{-}$beam the possible reaction mechanisms for $D^{-}$meson production at large $x_{F}$ and small $p_{T}$ (the soft regime) are illustrated in Fig. 1. 


\section{FIGURES}

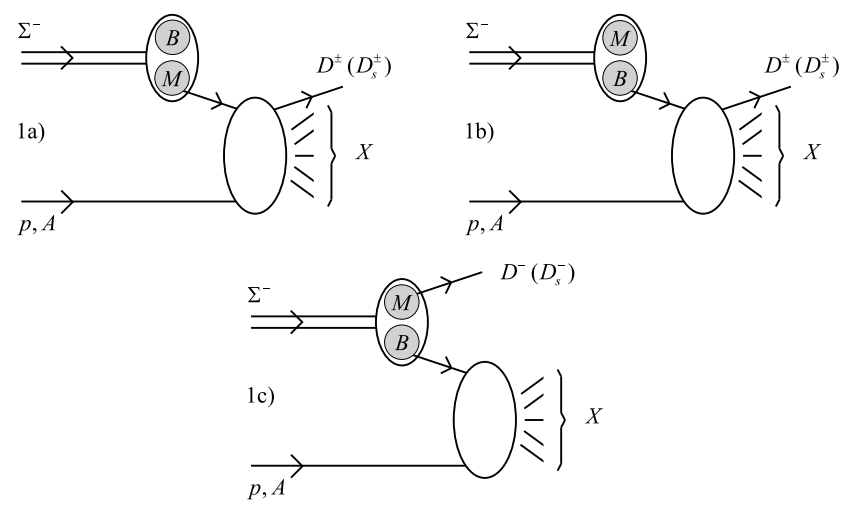

FIG. 1. $\Sigma^{-} p$ collision in which the projectile is in a $|M B\rangle$ state. Figs. 1a) and 1b) show the "indirect" $D^{ \pm}\left(D_{s}^{ \pm}\right)$production and 1c) the "direct" $D^{-}\left(D_{s}^{-}\right)$production.

In Fig. 1a the baryon just "flies through", whereas the corresponding meson interacts inelastically producing a $D$ meson in the final state. In Fig. 1b the meson just "flies through", whereas the corresponding baryon interacts inelastically producing a $D$ meson in the final state. In Fig. 1c the meson in the cloud is already a $D^{-}$(or $D_{s}^{-}$) which escapes (similar considerations hold for $D^{-}$production with a $\pi^{-}$beam). This last mechanism is responsible for generating asymmetries. We shall refer to the first two processes as "indirect production" $(I)$ and to the last one as "direct production" $(D)$. The first two are calculated with convolution formulas whereas the last one is given basically by the meson momentum distribution in the cloud initial $|M B\rangle$ state. Direct production has been widely used in the context of the MCM and applied to study $n, \Delta^{++}$and $\pi^{0}$ production [10]. Indirect meson production has been considered previously in [11].

Inside the baryon, in the $|M B\rangle$ state, the meson and baryon have fractional momentum $y_{M}$ and $y_{B}$ with distributions called $f_{M / M B}\left(y_{M}\right)$ and $f_{B / M B}\left(y_{B}\right)$ respectively (we shall use for them the short notation $f_{M}$ and $f_{B}$ ). Of course, by momentum conservation, $y_{M}+y_{B}=1$ and these distributions are related by $f_{M}(y)=f_{B}(1-y)$ [9.11]. The "splitting function" $f_{M}(y)$ represents the probability density to find a meson with momentum fraction $y$ of the total cloud state $|M B\rangle$. With $f_{M}$ and $f_{B}$ we can compute the differential cross section for production of $D$ mesons, which, in the reaction $\Sigma^{-} p \rightarrow D X$, is given by:

$$
\frac{d \sigma^{\Sigma^{-} p \rightarrow D X}}{d x_{F}}=\Phi_{0}+\Phi_{I}+\Phi_{D}
$$

where $\Phi_{0}$ and $\Phi_{I}$ refer respectively to "bare" and indirect contributions to $D$ meson production and $x_{F}$ is the fractional longitudinal momentum of the outgoing meson. $\Phi_{D}$ represents the direct process depicted in Fig. 1c and is given by [10,11]:

$$
\Phi_{D}=\frac{\pi}{x_{F}} f_{D}\left(x_{F}\right) \sigma^{\Xi}
$$

where $f_{D} \equiv f_{D^{-} / \Xi_{c}^{0} D^{-}}$and $\sigma^{\Xi}$ is the total $p \Xi_{c}^{0}$ cross section. An analogous expression can be written for the reaction $\pi^{-} p \rightarrow D X$. 
Using (3), we can compute the cross sections and also the leading $\left(D^{-}\right) / \operatorname{nonleading}\left(D^{+}\right)$ asymmetry:

$$
\begin{aligned}
A\left(x_{F}\right) & =\frac{\frac{d \sigma^{D^{-}}\left(x_{F}\right)}{d x_{F}}-\frac{d \sigma^{D^{+}}\left(x_{F}\right)}{d x_{F}}}{\frac{d \sigma^{D^{-}}\left(x_{F}\right)}{d x_{F}}+\frac{d \sigma^{D^{+}}\left(x_{F}\right)}{d x_{F}}} \\
& =\frac{\Phi_{D}+\Phi_{I}^{D^{-}}+\Phi_{0}^{D^{-}}-\Phi_{I}^{D^{+}}-\Phi_{0}^{D^{+}}}{\Phi_{D}+\Phi_{I}^{D^{-}}+\Phi_{0}^{D^{-}}+\Phi_{I}^{D^{+}}+\Phi_{0}^{D^{+}}} \\
& \simeq \frac{\Phi_{D}}{\Phi_{D}+2 \Phi_{I}^{D}+2 \Phi_{0}^{D}} \equiv \frac{\Phi_{D}}{\Phi_{T}}
\end{aligned}
$$

where the last line follows from assuming $\Phi_{I}^{D^{-}}=\Phi_{I}^{D^{+}}=\Phi_{I}^{D}$. This last assumption is made just for the sake of simplicity. In reality (and also in the calculations performed in [13]) these contributions are not equal and their difference is an additional source of asymmetry, which in some cases is not negligible. Since the "bare" states do not give origin to $D^{-} / D^{+}$asymmetries (they represent mostly perturbative QCD contributions which rarely leave quark pairs in the appropriate kinematic region), we have made use of $\Phi_{0}^{D+}=\Phi_{0}^{D-}=$ $\Phi_{0}^{D}$. The denominator of the above expression can be replaced by a parametrization of the experimental data:

$$
\Phi_{T}=\sigma_{0}\left[\left(1-x_{F}\right)^{n^{-}}+\left(1-x_{F}\right)^{n^{+}}\right]
$$

where $n^{-}$and $n^{+}$are powers used by the different collaborations to fit their data and $\sigma_{0} \simeq 4-7 \mu b$ as suggested by the data analysis performed in [1] 6 .

Inserting (44) and (6) into (5) the asymmetry becomes:

$$
A\left(x_{F}\right)=\frac{\pi \sigma^{\Xi}}{\sigma_{0}} \frac{f_{D}\left(x_{F}\right)}{x_{F}\left[\left(1-x_{F}\right)^{n^{-}}+\left(1-x_{F}\right)^{n^{+}}\right]}
$$

The behavior of (7) is controlled by $f_{D}\left(x_{F}\right)$. In the recent works with the MCM one finds two forms for the splitting functions. One comes from the evaluation of the relevant Feynman diagrams (Sullivan process) [9, 11] and the other comes from a light cone ansatz for the cloud state wave function [14]. We shall compute the asymmetries with both of them. The light cone splitting function is given by [14]:

$$
\begin{aligned}
f_{M}(y) & =\sum_{\lambda \lambda^{\prime}} \int_{0}^{\infty} \frac{d \mathbf{k}_{\perp}^{2}}{16 \pi^{2}}\left|\psi_{\lambda \lambda^{\prime}}\left(y, \mathbf{k}_{\perp}^{2}\right)\right|^{2} \\
& =\frac{1}{4 \pi^{2}} H^{2} \alpha^{2} y(1-y) \exp \left(-\frac{\mathcal{M}_{M}^{2}}{4 \alpha^{2}}\right)
\end{aligned}
$$

where $\psi_{\lambda \lambda^{\prime}}\left(y, \mathbf{k}_{\perp}^{2}\right)$ is the light cone wave function of the Fock state containing a meson $M$ (baryon $B$ ), with longitudinal momentum fraction $y$ (or $1-y$ ), transverse momentum $\mathbf{k}_{\perp}$ $\left(-\mathbf{k}_{\perp}\right)$ and helicity $\lambda\left(\lambda^{\prime}\right) ; \mathcal{M}_{M}^{2}=\frac{\mathbf{k}_{\perp}^{2}+m_{M}^{2}}{y}+\frac{\mathbf{k}_{\perp}^{2}+m_{B}^{2}}{1-y}$ is the invariant mass of the meson $(M)$ - baryon $(B)$ system for large longitudinal momenta, $m_{M}$ and $m_{B}$ are their masses, $H$ is a normalization constant and $\alpha$ is the width of the heavy meson-baryon state. 
Substituting (8) into (7) and remembering that in our case $y=x_{F}$, we can write our final expression for the asymmetry:

$$
\begin{aligned}
A_{1}\left(x_{F}\right) & =N_{1} \frac{\left(1-x_{F}\right)}{\left[\left(1-x_{F}\right)^{n^{-}}+\left(1-x_{F}\right)^{n^{+}}\right]} \\
& \times \exp \left[\frac{-1}{4 \alpha^{2}}\left(\frac{m_{D}^{2}}{x_{F}}+\frac{m_{\Xi}^{2}}{1-x_{F}}\right)\right]
\end{aligned}
$$

where $N_{1}=H^{2} \alpha^{2} \sigma^{\Xi} / 4 \pi \sigma_{0}$.

A striking feature of (9) is that in the limit $x_{F} \rightarrow 1$ it goes to zero regardless of the choice of two parameters $N_{1}$ and $\alpha$. This happens because when the leading meson (responsible for the asymmetry) has momentum one its accompanying cloud baryon must have momentum zero, being strongly virtual, thereby increasing the invariant mass of the cloud state and forcing this cloud configuration to have zero probability. For the pion beam, we obtain an analogous expression with the replacements $m_{\Xi} \rightarrow m_{D^{0 *}}, \sigma^{\Xi} \rightarrow \sigma^{D^{0 *}}$. In this case the parameters $H$ and $\alpha$ may assume different values. Apart from numerical changes, the qualitative behavior of $A_{1}$ remains the same, also for large $x_{F}$.

We now write the splitting function in the Sullivan process approach. The fractional momentum distribution of a pseudoscalar meson $M$ in the state $\mid M B^{\prime}>$ is given by [9, 11]:

$$
\begin{aligned}
f_{M}(y) & =\frac{g_{M B B^{\prime}}^{2}}{16 \pi^{2}} y \int_{-\infty}^{t_{\max }} d t \frac{\left[-t+\left(m_{B^{\prime}}-m_{B}\right)^{2}\right]}{\left[t-m_{M}^{2}\right]^{2}} \\
& \times F_{M B B^{\prime}}^{2}(t)
\end{aligned}
$$

where $t$ and $m_{M}$ are the four momentum square and the mass of the meson in the cloud state and $t_{\text {max }}=m_{B}^{2} y-m_{B^{\prime}}^{2} y /(1-y)$ is the maximum $t$, with $m_{B}$ and $m_{B^{\prime}}$ respectively the $B$ and $B^{\prime}$ masses. Following a phenomenological approach, we use for the baryon-meson-baryon form factor $F_{M B B^{\prime}}$, the exponential form:

$$
F_{M B B^{\prime}}(t)=\exp \left(\frac{t-m_{M}^{2}}{\Lambda_{M B B^{\prime}}^{2}}\right)
$$

where $\Lambda_{M B B^{\prime}}$ is the form factor cut-off parameter.

Considering the particular case where $B=\Sigma^{-}, B^{\prime}=\Xi_{c}^{0}$ and $M=D^{-}$, we insert (10) into (17) to obtain the final expression for the asymmetry in our second approach:

$$
\begin{aligned}
A_{2}\left(x_{F}\right) & =\frac{N_{2}}{\left[\left(1-x_{F}\right)^{n^{-}}+\left(1-x_{F}\right)^{n^{+}}\right.} \\
& \times \int_{-\infty}^{t_{\max }} d t \frac{\left[-t+\left(m_{\Xi}-m_{\Sigma}\right)^{2}\right]}{\left[t-m_{D}^{2}\right]^{2}} F_{D \Sigma \Xi}^{2}(t)
\end{aligned}
$$

where $N_{2}=g_{D \Sigma \Xi}^{2} \sigma^{\Xi} / 16 \pi \sigma_{0}$.

For the pion beam, we need also the Sullivan splitting function of the state $\mid \pi^{-}>\rightarrow$ $\left|D^{0 *} D^{-}\right\rangle$. In this state, the $D$ meson momentum distribution (which was computed in Ref. |13) turns out to be identical to (10) except for the bracket in the numerator which takes the form [13], $\left[-t+\left(\left(m_{\pi}^{2}-m_{D^{0 *}}^{2}-t\right) / 2 m_{D^{0 *}}\right)^{2}\right]$, and for trivial changes in the definitions, 
i.e., $g_{M B B^{\prime}}^{2} \rightarrow g_{\pi D D^{0 *}}^{2}, F_{M B B^{\prime}} \rightarrow F_{\pi D D^{0 *}}$ and $\Lambda_{M B B^{\prime}} \rightarrow \Lambda_{\pi D D^{0 *}}$. Realizing that $y=x_{F}$ in the above equations, we can see that in the limit $x_{F} \rightarrow 1, \quad t_{\max } \rightarrow-\infty$ and the integral in (12) goes to zero. In fact, it vanishes faster than the denominator and therefore $A_{2} \rightarrow 0$. This behavior does not depend on the cut-off parameter but it depends on the choice of the form factor. For a monopole form factor we may obtain asymmetries which grow even at very large $x_{F}$. Since $t$ controls the off-shellness of the emitted meson, which, in turn, is related to the virtuality of the $\mid M B^{\prime}>$ state (or $\mid M M^{\prime}>$ state in the case of the pion beam), the vanishing of $A_{2}$ happens for the same physical reason of the vanishing of $A_{1}$.

The $D_{s}^{-} / D_{s}^{+}$production asymmetry (with the $\Sigma^{-}$beam) can be calculated following the steps mentioned above and replacing the $\left|\Xi_{c}^{0} D^{-}\right\rangle$state by $\left|\Sigma_{c}^{0} D_{s}^{-}\right\rangle$. Of course, this implies different values for $\alpha$ and $N_{1}$ (in the light cone approach) and for $\Lambda$ and $N_{2}$ (in the Sullivan approach), but the qualitative discussion (and conclusions) presented above for $A_{1}$ and $A_{2}$ remain valid.

Before presenting our numerical results, we emphasize that i) our calculation is based on quite general and well established ideas, namely that hadron projectiles fluctuate into hadron-hadron (cloud) states and that these states interact with the target; ii) our results only depend on two parameters: $A_{1}$ depends on $\alpha$ and $N_{1}$ and $A_{2}$ depends on $\Lambda$ and $N_{2}$. Whereas $\alpha$ and $\Lambda$ affect the width and position of the maximum of the momentum distribution of the leading meson in the cloud (and consequently of the asymmetry), $N_{1}$ and $N_{2}$ are multiplicative constants which determine the strength of the asymmetry.

We show in Figs. 2a and 2b, respectively, our results for the asymmetries $D^{-} / D^{+}$and $D_{s}^{-} / D_{s}^{+}$(for a $\Sigma^{-}$beam). The results for a $\pi^{-}$beam are shown in Fig. 3. In all figures solid (dashed) lines represent $A_{2}\left(A_{1}\right)$. In both approaches we have two parameters which may be different from reaction to reaction. They are given in Tables 1 and 2 below:

\begin{tabular}{|c|c|c|c|c|c|}
\hline Eq. (9) & $\alpha(\mathrm{GeV})$ & $n^{+}$ & $n^{-}$ & $N_{1}$ & $\sigma^{D} / \sigma^{T}(\%)$ \\
\hline \hline$\Sigma^{-} A \rightarrow D^{-} X$ & 0.77 & 5.0 & 4.5 & 400.00 & 13.0 \\
\hline$\Sigma^{-} A \rightarrow D_{s}^{-} X$ & 0.47 & 7.0 & 4.0 & $0.72 \times 10^{9}$ & 9.0 \\
\hline$\pi^{-} A \rightarrow D^{-} X$ & 1.20 & 5.0 & 3.5 & 1.10 & 8.0 \\
\hline
\end{tabular}

Table 1. Parameters used in the asymmetry $A_{1}$

\begin{tabular}{|c|c|c|c|c|c|}
\hline Eq. (12) & $\Lambda(\mathrm{GeV})$ & $n^{+}$ & $n^{-}$ & $N_{2}$ & $\sigma^{D} / \sigma^{T}(\%)$ \\
\hline \hline$\Sigma^{-} A \rightarrow D^{-} X$ & 2.64 & 5.0 & 4.5 & 2.40 & 52.0 \\
\hline$\Sigma^{-} A \rightarrow D_{s}^{-} X$ & 2.52 & 7.0 & 4.0 & 5.20 & 67.0 \\
\hline$\pi^{-} A \rightarrow D^{-} X$ & 2.88 & 5.0 & 3.5 & 0.82 & 20.0 \\
\hline
\end{tabular}

Table 2. Parameters used in the asymmetry $A_{2}$ 

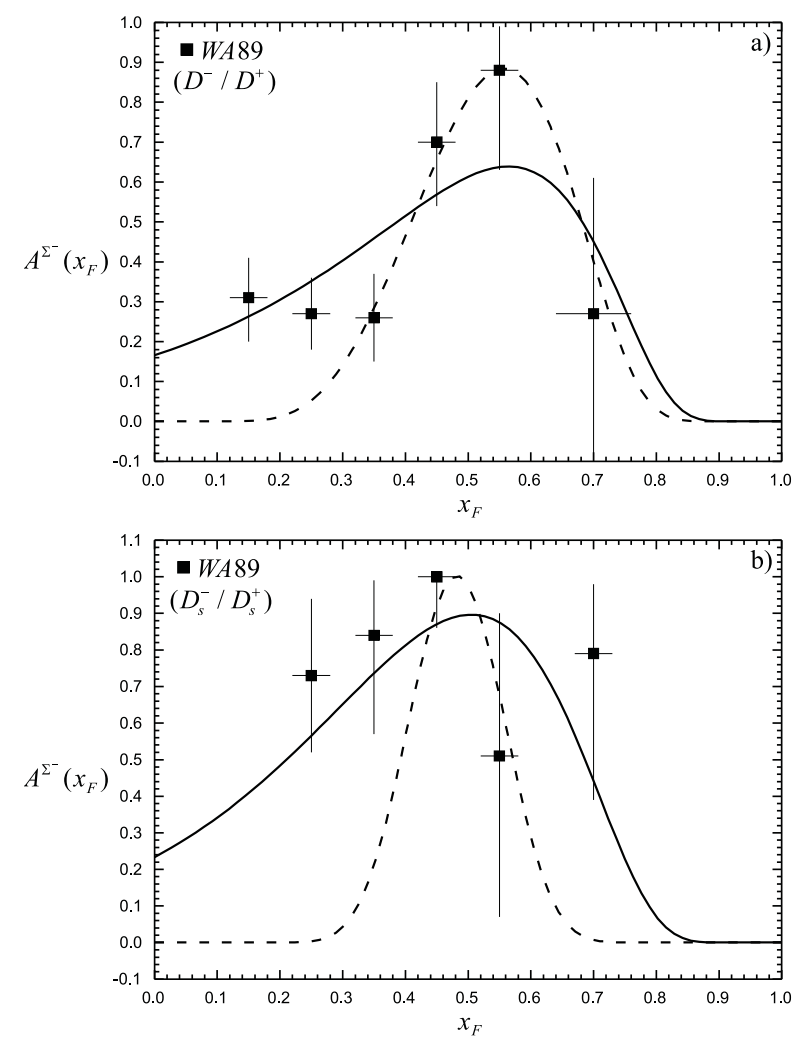

FIG. 2. (2a) Comparison of the MCM asymmetry, Eq. (7), with experimental data [0] for $D^{-} / D^{+} ;(2 \mathrm{~b})$ the same as $(2 \mathrm{a})$ for $D_{s}^{-} / D_{s}^{+}$.

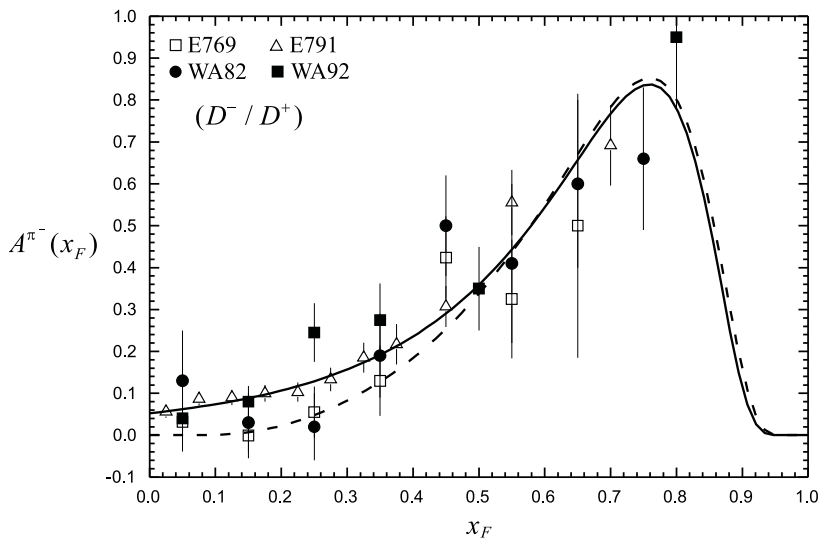

FIG. 3. Comparison of the MCM asymmetry, Eq. (7), with experimental data 迎顿 for $D^{-} / D^{+}$.

As it can be seen in Figs. 2 and 3 the agreement between the MCM and data is very good. In our picture it is simple to understand why the $D^{-} / D^{+}$asymmetry peaks at $x_{F} \simeq 0.55$ for the $\Sigma^{-}$beam (Fig. 2a) whereas it peaks at a much larger value $x_{F} \simeq 0.8$ for the $\pi^{-}$ beam (Fig. 3). The $D^{-}$meson in the $\Sigma^{-}$beam originates from the $\left|\Xi_{c}^{0} D^{-}\right\rangle$state and in the $\pi^{-}$beam it comes from the $\left|D^{0 *} D^{-}\right\rangle$state. Since in the meson-meson state the masses are closer than they are in the baryon-meson state, the $D^{-}$is "faster" inside the pion than 
inside the $\Sigma^{-}$. In (7),$f_{D}\left(x_{F}\right)$ will peak sooner and die faster for the $\Sigma^{-}$. We emphasize that what shifts the peaks of the asymmetries are the masses involved rather than the $\alpha$ (or $\Lambda)$ parameter. This makes the overall behavior of the asymmetries weakly dependent on parameter choices.

The last column in both tables shows the ratio between the direct and total $D^{-}$cross sections (where $\sigma^{T}=\sigma_{0} \int d x_{F}\left(1-x_{F}\right)^{n^{-}}$). We find this quantity quite model dependent. This is also a consistency check. In order to treat the cloud as a perturbation we expect this ratio to be at the level of $10-20 \%$. In two cases, we observe a significant deviation from this expectation. This means that, in these cases, we need a large normalization constant for the cloud state responsible for direct production (which implies large $N_{2}$ ), in order to reproduce the observed asymmetry. This is a consequence of neglecting the asymmetry generated by indirect production, i.e., in these cases, the approximation $\Phi_{I}^{D^{-}}=\Phi_{I}^{D^{+}}$is not a good one. A more complete discussion, taking this fact into account is presented in [13. Here, for the sake of the argument, we prefer to keep the calculation simple and keep our fits as they are, being implicit that the large $N_{2}$ 's mimic the inclusion of indirect production.

In conclusion, we have shown that the MCM provides a good understanding of the charm production asymmetries in terms of a simple physical picture with few parameters. It connects the behavior of the asymmetries at large $x_{F}$ with the charm meson momentum distribution within the cloud state. We can explain why we observe asymmetries, why they are different for different beams and we are led to the conclusion that they may vanish at very large $x_{F}$. This approach can easily be extendend to charm baryon production and also to $B$ production [13].

\section{ACKNOWLEDGMENTS}

This work has been supported by CNPq and FAPESP, under contract number 2000/04422-7. 


\section{REFERENCES}

[1] M. I. Adamovich et al., (WA82 Collab.), Phys. Lett. B306, 402 (1993).

[2] G. A. Alves et al., (E769 Collab.), Phys. Rev. Lett. 77, 2392 (1996).

[3] E. M. Aitala et al., (E791 Collab.), Phys. Lett. B411, 230 (1997).

[4] M. I. Adamovich et al., (WA92 Collab.), Nucl. Phys. B495, 3 (1997).

[5] M. I. Adamovich et al., (WA89 Collab.), Eur. Phys. J. C8, 593 (1999); C13, 247 (2000).

[6] M. Iori et al., (SELEX Collab.), Nucl. Phys. B (Proc. Suppl.) 75 B, 16 (1999); hepex/0009049; J.C. Anjos and E. Cuautle, hep-ph/0005057.

[7] O. I. Piskounova, Nucl. Phys. Proc. Suppl. 50 (1996) 179; C.E. Aguiar, T. Kodama, R.A.M.S. Nazareth, G. Pech, Phys. Rev. C53, 448 (1996); F. O. Durães, F. S. Navarra, C. A. A. Nunes and G. Wilk, Phys. Rev. D53, 6136 (1996); J. Dias de Deus and F. O. Durães, Eur. Phys. J. C13, 647 (2000).

[8] T. Gutierrez and R. Vogt, Nucl. Phys. B539, 189 (1999) and references therein; R. Vogt and S. J. Brodsky, Nucl. Phys. B438, 261 (1995).

[9] for a review see J. Speth and A. W. Thomas, Adv. Nucl. Phys. 24, 83 (1998); S. Kumano, Phys. Rep. 303, 183 (1998); A.W. Thomas, Phys. Lett. B126, 97 (1983).

[10] H. Holtmann, A. Szczurek and J. Speth, Nucl. Phys. A569,631 (1996); N. N. Nikolaev, W. Schaefer, A. Szczurek and J. Speth, Phys. Rev. D60, 014004 (1999).

[11] F. Carvalho, F. O. Durães, F. S. Navarra and M. Nielsen, Phys. Rev. D60, 094015 (1999); F. S. Navarra, M. Nielsen and S. Paiva, Phys. Rev. D56, 3041 (1997); F. Carvalho, F. O. Durães, F. S. Navarra and M. Nielsen, and F.M. Steffens, Eur. Phys. J. C18 127 (2000).

[12] F. S. Navarra, M. Nielsen, C. A. A. Nunes and M. Teixeira, Phys. Rev. D54, 842 (1996); S. Paiva, M. Nielsen, F. S. Navarra, F. O. Durães and L. L. Barz, Mod. Phys. Lett. A13, 2715 (1998); W. Melnitchouk and A.W. Thomas, Phys. Lett. B414, 134 (1997).

[13] F. Carvalho, F. O. Durães, F. S. Navarra and M. Nielsen, to be submitted to Phys. Rev. D.

[14] S. J. Brodsky and B.-Q. Ma, Phys. Lett. B381, 317 (1996); F.G. Cao and A.I. Signal, Phys. Rev. D60, 074021 (1999). 